\title{
THE BOLD AND THE BEAUTIFUL: HOW ASPECTS OF PERSONALITY AFFECT FOREIGN LANGUAGE PRONUNCIATION
}

\author{
MARTIN HINTON \\ University of Łódź \\ mdhinton@tlen.pl
}

\begin{abstract}
This paper reports on a study into the inter-relationships amongst foreign language pronunciation, mimicry ability and a range of personality and attitudinal factors. It will begin with a brief review of studies into affective influences on pronunciation ability (Arnold 1999, Hu \& Reiterer 2009) and research into the importance of mimicry talent (Jilka 2009; Piske, MacKay \& Flege 2001). This will be followed by a short description of a pilot study carried out prior to the main experiment.

In the main study, a group of Polish learners of English completed a number of mimicry tasks in three languages: Italian, Dutch and Chinese, as well as a narration task in English. Mimicry performance and English pronunciation were then assessed by native speakers and compared. Participants also completed a questionnaire concerning their feelings about the languages they were to mimic and a second questionnaire designed to detect affective factors such as language learning anxiety, as well as attitudes towards the pronunciation of Polish and English. The pilot study suggested that the perceived attractiveness of the foreign language to be mimicked did not affect the performance of most participants, and that mimicry skill was fairly constant across languages. However, those who were particularly concerned about their personal appearance showed greater fluctuation in their ability to mimic and their performance appeared to be influenced by their attitude towards the language. This is referred to by the author as the Cecily effect. That study also confirmed the results of my previous experimental work showing that mimicry skill is correlated to some degree with English language pronunciation and that both pronunciation and mimicry are negatively affected by high levels of anxiety. The main study sets out to investigate whether or not these conclusions hold true for a larger sample population and also seeks to determine the effect of confidence and willingness to take risks on scores for both foreign language pronunciation and mimicry exercises.
\end{abstract}

Keywords: mimicry, pronunuciation, affect, Cecily effect

\section{Introduction}

Foreign language pronunciation, like all other aspects of linguistic performance, is influenced by a range of variables, of which each individual speaker has a unique combination. Some of those factors are related to the learning environment the speaker has enjoyed, others are more personal and, therefore, very hard for the teacher, and often 
the student himself, to control. This second group includes cognitive abilities, personality features and attitudes towards the language, as well as the great affective forces of motivation and anxiety. Indeed, pronunciation might well be thought particularly susceptible to affective and emotional factors, since it is the first thing about our speech in a foreign tongue to be judged by those who hear us. The speaker's capacity to handle complex grammatical structures or use appropriate vocabulary is not readily apparent in his first utterance, but his pronunciation, and specifically his accent, is. In addition to being judged by others, the actual quality of the sounds we are making when speaking foreign tongues is also particularly relevant to our self-image and likely to bring fears that one may be sounding foolish before one has really said anything very much at all.

There are two very good reasons to study these effects: firstly, looking at the cognitive processes involved and how they may or may not be influenced by other factors may lead to a properly understood concept of foreign language pronunciation aptitude, for which tests might be developed to predict which learners have the potential to go on to native-like levels of pronunciation and to detect which were likely to need special assistance in the same area. Secondly, the study of how attitudes towards particular languages and sound systems influence learners' ability to pronounce them will assist teachers in identifying reasons for weaknesses in their students and give them the opportunity to attempt to modify those attitudes in a favourable direction. It should be noted too, that where it is not possible to modify the student, it may be possible to modify the material and the approach.

Throughout this paper it is assumed that learners of foreign language should, and to a large extent actually do, aim at a standard pronunciation model approved by the native speaker community. Due to the prevailing preference of teachers and institutions in Poland, this is taken to be a standard British accent generally referred to as Received Pronunciation (RP) for the purposes of assessment. It is accepted, however, that this assumption is somewhat controversial and, indeed, the degree to which native-like accent is in fact striven for is something which the study tools try to capture.

\section{Factors affecting pronunciation}

This section will provide a very brief review of some of the factors which have previously been identified as relevant to the quality of pronunciation and pay particular attention to those with which the study itself is concerned. In their exhaustive review of work done at that time Piske, MacKay and Flege (2001) list several factors which had been claimed to affect pronunciation in learners. They were: age of learning, length of residence in target language community, gender, degree of formal instruction, motivation, and language learning aptitude.

The impact of age of learning is generally associated with the Critical Period Hypothesis $(\mathrm{CPH})$ which states that after a certain point, children lose the ability to develop accent-free speech in a new language. This theory suffers from the existence of a great many versions, some with more than one cut-off point, many citing different ages, spanning the entire length of childhood. While this variety does not necessarily mean the theory is incorrect: there is no doubt that 'early learners speak L2 with a lower 
degree of foreign accent than late learners' (Piske, Mackay \& Flege 2001: 197), it has led critics such as David Singleton to cast doubt on it. He points out that as well as different ages at which the critical period is supposed to finish, there are as many as three distinct varieties of explanation for the phenomenon: neuro-biological, cognitive-developmental, and affective-motivational. Rather than trying to argue against it, therefore, he simply notes that 'it is like the mythical hydra, whose multiplicity of heads and capacity to produce new heads rendered it impossible to deal with' (Singleton 2005: 280). At any rate, the lack of direct correspondence between age of learning and pronunciation level makes it clear that, while older learners are far less likely to achieve high levels of proficiency in pronunciation, other factors are at play.

Length of residence is, obviously, only of relevance in natural, second language settings, and the results of studies have been mixed, with the actual use made of the language a possibly decisive factor. This is also the case with gender: where it has been found to be a factor, females have been rated more highly for accent, but not all studies have seen a significant difference. Piske et al. are also unconvinced by the influence of formal instruction on foreign accentedness concluding 'there is little evidence to date that amount of formal instruction as such affects degree of L2 foreign accent' (2001: 201), although this would certainly be disputed by, among others, Derwing and Rossiter (2003) and Graeme Couper (2003, 2006).

Of the factors mentioned so far, only gender will be looked at in this study: the learning experiences of the participants, in terms of age at which learning began and type of instruction they have received, are very similar as they are from a homogeneous age and social group. The two remaining factors, however, are of much greater interest here. Motivation is the only affective factor on the list and to some extent, therefore, stands proxy for affect in general. Piske et al. point out that while motivation has generally been found to have a role in determining pronunciation outcomes, there are problems with the quantification of it in many studies. More recently, Małgorzata Baran-Łucarz (2013) has investigated the effect of anxiety on the learning of phonetics. She points out that although a number of more specific anxieties have been identified since the concept of foreign language classroom anxiety (Horwitz, Horwitz \& Cope 1986) was accepted, 'so far no instrument has been designed to examine specifically the nature of pronunciation anxiety, which could address feelings evoked by the way one sounds or looks like when talking in a FL' (Baran-Łucarz 2013: 60-61). The application of her own attempt to construct such a tool leads her to the conclusion that what she refers to as Phonetics Learning Anxiety 'is indeed an important factor determining success in FL pronunciation learning' (2013: 74). This statement must be subject to the usual questions over the direction of the anxiety/performance relationship (Ganschow \& Sparks 1996) but represents an interesting step in the understanding of the impact of affective factors on pronunciation.

The final factor mentioned by Piske et al. is that of language learning aptitude. It is important to note that they are not referring to the Carrollian concept (Carroll, 1965), as tested by the Modern Languages Aptitude Test, but rather to innate factors in general: specifically, they discuss musical ability and mimicry. Studies into musical ability have had mixed results, partly because that ability can have various definitions, and few conclusions can be drawn. Mimicry, on the other hand, 'has repeatedly been identified as a significant and independent predictor of degree of L2 foreign accent' (Piske et al. 2001: 
202). They refer in particular to a well-known study by Purcell and Suter (1980) which was actually a reassessment of Suter's earlier work, and found only L1 to have a greater influence on accentedness than mimicry skill. A similar pattern has also been found repeatedly in my own work (Hinton 2013).

A recent large-scale study into a range of aptitudinal and affective/psychological factors influencing pronunciation is reported in a book edited by Grzegorz Dogil (2009) and a number of follow-up papers by his team. Using a battery of mimicry (in Hindi) and English pronunciation tests on a large group of German learners, the team divided their subjects into groups of high pronunciation talent, average pronunciation talent and low pronunciation talent. When representatives of each group were subjected to magnetic resonance (MR) brain scanning whilst performing a mimicry task, clear differences were recorded between the talented and untalented participants, leading the researchers to conclude that they had found evidence of 'a distinct neurofunctional/neuro-anatomical signature of speech imitation ability' (Reiterer et al. 2011: 9). This kind of analysis is a vast improvement on vague concepts such as having 'a good ear', traditionally associated with mimicry.

Analysis of the personalities of the same group of German learners of English, however, produced far less clear-cut results. Despite the employment of an extremely wide range of psychological perspectives and tools for measurement, the researchers found only a weak link between pronunciation talent and empathy and an increase in positive affects amongst the more talented when performing phonetic-articulation tasks (Hu \& Reiterer 2009: 119). It appears from this study that general personality traits per se do not have great influence on pronunciation ability and that any search for links between the individual's attitudes and character, and degree of foreign accent will have to concentrate on attitudes and beliefs more specific to the target language and its sound.

One further complicating factor is the degree to which differing variables of cognitive functioning, motivation and anxiety, and attitudinal or personality attributes may interact. This continued interaction and interdependence has led Zoltan Dornyei to speak of the view of stable, quantifiable individual differences as a 'myth'. He believes that this traditional conception 'does not do justice to the dynamic, fluid and continuously fluctuating nature of learner factors and neither does it account for the complex internal and external interactions that we can observe' (Dornyei 2010: 253). There is no room here to examine what all of those interactions might be or even to discuss which are of most relevance to the study of pronunciation performance, however, the possibility of identifying some key factors and how they affect each other as well as eventual learner outcomes is part of the motivation for this study and is described more fully in the section below which details the results of the pilot study done in preparation for the larger-scale experiment which is the focus of the paper.

\section{The pilot study}

The motivation behind the present study was a set of anomalous results in earlier work which had used mimicry to assess pronunciation talent as part of a wider investigation into language learning aptitude (Hinton 2012). The results gave the impression that while there was a broad correlation between mimicry skill and pronunciation in English, 
certain individuals appeared to under-perform in mimicry tasks involving an exotic language. Further investigation of these individuals showed them all to be wellpresented females who obviously took considerable care over their appearance. The possibility that vanity or an anxiety over appearance might have affected the mimicry results led to the suggestion that a Cecily effect, (after the Oscar Wilde character who felt ugly after speaking German) might be in operation. The original intention, then, of this study was to seek further evidence of that effect. A pilot study was conducted on just 10 participants in order to test the tools and determine whether or not the same effect would be reproduced on the original subjects. A full description and assessment of the tools and a discussion of the results has been provided elsewhere (Hinton in press) but below is a brief summary of the key points.

- Although the population was too small for statistical significance, correlations between mimicry and accent $(\mathrm{r}=0.42)$, and mimicry and anxiety $(\mathrm{r}=-0.35)$ were around the expected levels.

- No correlation was found between perceived attractiveness of the language and mimicry performance. However, the three participants with the highest interest in their appearance all did relatively worse than the other 7 on the least attractive language (Chinese).

- This under-performance gives greater plausibility to the existence of the Cecily effect among a proportion of the population.

- The tools performed reasonably well, but certain changes were identified as necessary for the main study.

Perhaps the key conceptual addition for the main study was the introduction of a scale for boldness. While anxiety clearly exerted a negative influence on mimicry and pronunciation scores, there was a suspicion that the mimicry scores of certain individuals were being boosted by their boldness or willingness to take risks and the main study set out to examine whether this was in fact the case and whether that boldness also had an effect on degree of accent.

\section{An experiment}

The aim of the practical study undertaken, then, was to test the following hypotheses:

1 - That for the section of the population with very high interest in their appearance, mimicry performance would be affected by feelings about the attractiveness of the input language. (The Cecily effect).

2 - That high boldness would lead to improved performance in mimicry but not in foreign language pronunciation.

These hypotheses were to be tested by assessing certain affective variables and comparing their levels in each individual with that individual's performance on a series of mimicry tests and an English accent assessment exercise, as described below. 


\section{Method}

The experiment was conducted in the autumn of 2013 at the foreign language teacher training college in Sieradz, Poland. There were 31 participants in total: 6 males and 25 females; this imbalance reflecting the overall proportions in the college population, not chosen by design. The age range of the participants was from 17 to 24.29 of the participants were students of the college at the time of the study (aged 19 to 22), one had recently graduated and one was a high school student. The graduate was included as she had previously been identified as possessing a particular talent for pronunciation. The youngest participant was considered to have a similar level of English to the college students, had had significant exposure to a native English accent in a foreign language setting, and had previously expressed high interest in her appearance.

The study was divided into three steps. First, the participants were asked to complete a survey which consisted of 35 statements to be rated on a 5-point Likert scale, from 1 completely disagree to 5 - completely agree. The statements were grouped into 7 categories of 5 questions each, dispersed randomly throughout the survey. The categories which the statements were intended to assess were as follows: importance of sound, importance of appearance, language learning anxiety, regard for Polish, regard for English, openness to foreign cultures, and boldness. The first six of these categories were trialled in the pilot study (see section 3 above) and the seventh, boldness, was added as a result of the apparent influence that a willingness to take risks in earlier mimicry tests had had on the inflated scores of some participants. A full list of statements is given in appendix A. The scores given to the 5 statements in each category (some reversemarked) were summed to give a score out of 25 for that variable, for each individual. It should be noted that no measure for motivation was included because in previous experiments all participants had claimed to be highly motivated, and perhaps believed their answers to be truthful. It is hoped, therefore, that other variables, such as importance of sound and regard for English, may stand proxy for a scale of pronunciation motivation.

The second part of the test made use of three input languages: Italian, Mandarin Chinese and Dutch. None of the participants had any experience of learning or working with these languages, although, obviously, the degree of familiarity with each would be expected to vary from individual to individual. The choice of languages was based on an informal survey of students which suggested that Romance languages were the most pleasing to the ear and exotic Asian languages the least. Many respondents also cited German as an unpleasant language. The use of German was not possible since the participants had all spent some time learning that language, with varying degrees of success. However, Dutch was considered to be an intriguing substitute, being a close relative of German without the historical baggage which might be expected to influence Polish speakers in their opinion of its sound. The choice of all three languages was also influenced by the availability of a native speaker to assist with the grading.

At first, participants were played a sound recording taken from a popular television programme in each of the three languages. Each recording featured a male and a female in fluent discussion and lasted around 30 seconds. After each clip, participants were asked to complete a short questionnaire, featuring 4 statements (see appendix B) on a 5point Likert scale, to measure how attractive they found the sound of the language. They 
were also asked to try and identify the language if possible, but were not told immediately if they were correct.

This procedure was followed directly by a series of 3 mimicry tests, one in each of the input languages, featuring 12 words or short phrases. These were either recorded by the native speaker graders or taken from Google Translate sound clips. Participants heard each word or phrase once and repeated it immediately to the best of their ability. These responses were recorded for later marking. The marking system adopted had been used in the pilot study and other mimicry tests I had previously conducted and found to be simple and effective. Each response was graded on a scale of $0-3$, where $0=$ no response, 1 = a poor effort, $2=$ a reasonable effort and $3=$ a good effort. Although it could be argued, quite successfully, that 'poor', 'reasonable' and 'good' are subjective terms which cannot possibly be interpreted in the same way by each assessor, that would be to miss the point. The absolute score for each test is not important since no attempt was made to standardise the level of difficulty for each language other than to keep the number of syllables similar: this study did not intend to investigate which languages are more difficult to mimic. What is important is the relative strength of each participant within the group for each language compared to the relative strength of his accent and relative importance of certain other variables. What matters, therefore, in the marking process is only that each assessor is consistent across the 31 participants. Total consistency, of course, is not possible and borderline cases between a 1 and a 2 or a 2 and a 3 may receive a different mark at different times, but, since the responses are recorded, the assessor may listen as many times as necessary to each participant to be sure of fairness. Other problems, however, did appear as a result of this marking scheme and are discussed in the results section below. The scores given below for each language are those of the native speaker assessor.

The third and final part of the test was an exercise in English narration. Each participant was asked to read aloud a passage after looking at it briefly. The passage (see appendix C) was taken from an unpublished story written by the researcher so as to ensure that it was equally unknown to all participants. The recordings were then assessed for quality of English accent by two native speakers of English, one male and one female. Each participant received a mark out of 10 for accent: mistakes in pronunciation and stress due to unfamiliarity with certain words were ignored. The scores given in the results below are an average of the marks of the two assessors.

\section{Results and discussion}

The various experimental tools produced a large amount of data on each participant. These data are summarised in the following tables and discussed in turn. The number of participants is constant at 31 throughout all the indices. The first table gives information about the results of the survey. The maximum score in each category is 25 , the minimum 5 . 


\begin{tabular}{|c|c|c|c|c|c|c|c|}
\hline & Sound & Appearance & Anxiety & $\begin{array}{c}\text { Openness } \\
\text { to cultures }\end{array}$ & $\begin{array}{c}\text { Regard } \\
\text { for } \\
\text { Polish }\end{array}$ & $\begin{array}{c}\text { Regard } \\
\text { for } \\
\text { English }\end{array}$ & Boldness \\
\hline Mean & 19.06 & 17.03 & 16.94 & 18.87 & 16.90 & 18.19 & 16.23 \\
\hline $\begin{array}{c}\text { Standard } \\
\text { deviation }\end{array}$ & 3.29 & 3.72 & 2.26 & 3.91 & 2.26 & 2.89 & 2.89 \\
\hline High & 24 & 24 & 22 & 24 & 20 & 24 & 22 \\
\hline Low & 10 & 8 & 10 & 9 & 12 & 12 & 11 \\
\hline
\end{tabular}

Table 1: Survey - general information

The highs and lows shown in the table make it clear that the categories provoked wideranging responses. The higher standard deviations for interest in appearance and openness to other cultures illustrate that these categories produced the most varied responses.

Table 2 gives the same information for the second part of the survey, dealing with the attractiveness of the input languages. The four statements given to participants are listed in appendix B. However, responses to the fourth statement are not included in the results. A similar statement proved troublesome in the pilot study and despite a small change to the wording, again appeared to cause confusion with some participants unsure whether 'sounds funny' was a positive or negative opinion. The scores for each language, therefore, have a maximum of 15 and a minimum of 3.

\begin{tabular}{|c|c|c|c|c|}
\hline & Italian & Chinese & Dutch & Total \\
\hline Mean & 11.29 & 7.52 & 8.65 & 27.45 \\
\hline Standard deviation & 2.64 & 3.15 & 3.33 & 5.23 \\
\hline High & 15 & 15 & 15 & 37 \\
\hline Low & 5 & 3 & 3 & 14 \\
\hline Correlation with openness & 0.1 & 0.54 & 0.05 & 0.41 \\
\hline Correlation with anxiety & 0.15 & -0.44 & 0.22 & -0.05 \\
\hline
\end{tabular}

Table 2: Attractiveness of languages survey

This table makes it clear that Italian was, as expected, the most popular language with the sound of Chinese least attractive. At least one person gave maximum points to each of the languages, but only Italian managed to avoid a minimum score. This enormous difference in taste makes clear the potential for feelings about sound to be a significant variable in performance across languages. While these differences in feelings may have been expected, the correlations with the variables from the affect survey make for interesting reading. While neither openness to foreign cultures nor anxiety had much 
effect on feelings about the European languages, the correlations with scores for Chinese are significant $(\mathrm{p}<0.014)$.

Table 3 provides a summary of the performance statistics. In this table the mimicry scores for each language, out of a maximum of 36, are those of the native speaker graders, and the total the sum of those grades. The number of $3 \mathrm{~s}$, the highest mark, awarded to each participant, however, is based on the assessment of the researcher as other graders did not listen to all languages and there were wide differences among them in the degree of generosity in their marking which could distort the figure. The reason for the inclusion of this measure will become clear below.

\begin{tabular}{|c|c|c|c|c|c|c|}
\hline & $\begin{array}{c}\text { Italian } \\
\text { mimicry }\end{array}$ & $\begin{array}{c}\text { Chinese } \\
\text { mimicry }\end{array}$ & $\begin{array}{c}\text { Dutch } \\
\text { mimicry }\end{array}$ & $\begin{array}{c}\text { Total } \\
\text { mimicry }\end{array}$ & $\begin{array}{c}\text { Total } \\
3 \mathrm{~s}\end{array}$ & $\begin{array}{c}\text { English } \\
\text { accent }\end{array}$ \\
\hline Mean & 22.97 & 24.45 & 21.87 & 69.29 & 8.52 & 5.48 \\
\hline $\begin{array}{c}\text { Standard } \\
\text { deviation }\end{array}$ & 3.55 & 6.00 & 4.54 & 11.05 & 4.15 & 1.32 \\
\hline High & 29 & 33 & 30 & 87 & 17 & 9.5 \\
\hline Low & 16 & 12 & 13 & 48 & 3 & 3.5 \\
\hline
\end{tabular}

Table 3: Summary of performance scores

Interesting points in this table are the low mean and 'high' scores for Italian. Judging from the researcher's own grades (means: 26.68, 20.45, 15.65) and discussions with participants after the tests, there is little doubt that the Italian test was the easiest and the Dutch the hardest for the Poles taking them. The Italian grader reflects this by giving a lowest mark of 16 but is clearly very demanding at the higher end of the scale, expecting a very good Italian accent where the other graders were satisfied with something rather less. This trend is also reflected in the low standard deviation of his results. For this reason, the Italian mimicry score can be said to be less useful than the others in determining differences in mimicry skill, since so many of the responses were grouped together as reasonable but not good.

One more point to note is that the accent score may seem low by normal standards at a mean of 5.48. It is true that the weakness of the participants' language skills did prove to be an obstacle in the grading of the accents but the graders were instructed to try to use the full range of scores from 1 to 10 and not group all grades around 5 to 8 as is often the case when marking out of 10 . Taking this into account, a mean in the middle of the range at 5.48 , is to be expected.

Table 4 below shows correlations between the survey categories and the performance measures.

\begin{tabular}{|c|c|c|c|c|c|c|}
\hline & $\begin{array}{c}\text { Italian } \\
\text { mimicry }\end{array}$ & $\begin{array}{c}\text { Chinese } \\
\text { mimicry }\end{array}$ & $\begin{array}{c}\text { Dutch } \\
\text { mimicry }\end{array}$ & $\begin{array}{c}\text { Total } \\
\text { mimicry }\end{array}$ & $\begin{array}{c}\text { Total } \\
3 \mathrm{~s}\end{array}$ & $\begin{array}{c}\text { English } \\
\text { accent }\end{array}$ \\
\hline Sound & -0.01 & 0.12 & 0.21 & 0.15 & 0.17 & 0.14 \\
\hline Appearance & 0.29 & -0.09 & 0.13 & 0.10 & 0.18 & 0.21 \\
\hline
\end{tabular}




\begin{tabular}{|c|c|c|c|c|c|c|}
\hline & $\begin{array}{c}\text { Italian } \\
\text { mimicry }\end{array}$ & $\begin{array}{c}\text { Chinese } \\
\text { mimicry }\end{array}$ & $\begin{array}{c}\text { Dutch } \\
\text { mimicry }\end{array}$ & $\begin{array}{c}\text { Total } \\
\text { mimicry }\end{array}$ & $\begin{array}{c}\text { Total } \\
3 \mathrm{~s}\end{array}$ & $\begin{array}{c}\text { English } \\
\text { accent }\end{array}$ \\
\hline Anxiety & -0.21 & -0.24 & -0.11 & -0.24 & -0.19 & 0.18 \\
\hline $\begin{array}{c}\text { Openness to } \\
\text { cultures }\end{array}$ & 0.21 & 0.07 & 0.30 & 0.23 & -0.01 & -0.20 \\
\hline $\begin{array}{c}\text { Regard for } \\
\text { Polish }\end{array}$ & -0.06 & 0.13 & 0.18 & 0.12 & 0.31 & 0.09 \\
\hline $\begin{array}{c}\text { Regard for } \\
\text { English }\end{array}$ & 0.20 & 0.45 & 0.44 & 0.49 & 0.41 & 0.15 \\
\hline Boldness & 0.52 & 0.41 & 0.43 & 0.57 & 0.43 & 0.14 \\
\hline $\begin{array}{c}\text { Fondness for } \\
\text { Italian }\end{array}$ & 0.20 & -0.16 & 0.16 & 0.04 & 0.11 & 0.37 \\
\hline $\begin{array}{c}\text { Fondness for } \\
\text { Chinese }\end{array}$ & 0.11 & 0.24 & 0.41 & 0.33 & 0.07 & -0.03 \\
\hline $\begin{array}{c}\text { Fondness for } \\
\text { Dutch }\end{array}$ & -0.08 & -0.09 & 0.18 & 0.00 & 0.02 & 0.09 \\
\hline Total fondness & 0.11 & -0.01 & 0.44 & 0.22 & 0.11 & 0.23 \\
\hline English accent & 0.04 & 0.09 & 0.12 & 0.12 & 0.37 & \\
\hline
\end{tabular}

$$
\begin{aligned}
& \mathrm{df}=29 \\
& \mathrm{p}<0.05 \quad \text { rcrit. }=0.31 \\
& \mathrm{p}<0.01 \quad \text { rcrit. }=0.42 \\
& \text { (one-tailed test) }
\end{aligned}
$$

Table 4: Affect/performance correlations

To summarise briefly, the importance of sound variable shows only very weak relationships with other factors. Interest in appearance, however, would certainly have had a more significant correlation with the overall mimicry score if it had not been for the low correlation with Chinese, rated the least attractive of the input languages, this is caused by relatively poor performance in Chinese by those with high scores for appearance, something which will be discussed again below. Anxiety, as expected, correlates negatively, if only weakly, with all measures of mimicry but, surprisingly does not have a negative correlation with accent. Openness to foreign cultures reaches a correlation of 0.28 (significant at $\mathrm{p}<0.06$ ) with total mimicry but not with English accent, while regard for Polish does not seem to influence either. The high correlation between regard for English and mimicry scores, but far weaker relationship with accent is hard to explain, but could possibly be down to a willingness to please the researcher, by praising his language and doing as well as possible at the mimicry task. Such effects are a constant difficulty in studies of this nature, especially where the participants are also students of the researcher. 
Perhaps the clearest relationship seen in the table is the correlation between boldness and mimicry score. This is significant across all the measures, reaching 0.57 (significant at $\mathrm{p}<0.00045$ ) for the total score. There is no matching correlation, however, with accent, suggesting that boldness causes a large amount of interference when attempting to predict foreign accent levels from mimicry scores. These data appear to support hypothesis 2 in section 4 .

All three languages show a degree of correlation between fondness for their sound and mimicry performance in that language $(0.20,0.24,0.18)$ and the totals correlate at 0.22 (significant at $\mathrm{p}<0.12$ ). The correlation between English accent and total mimicry skill is surprisingly weak at just 0.12 . Interestingly though, the correlation between good mimicry responses, the number of $3 \mathrm{~s}$ scored, and accent is much higher at 0.37 (significant at $\mathrm{p}<0.02$ ). This difference suggests that mimicry tests should be designed in such a way as to only reward the best examples of mimicry and not give points for poor attempts which reflect only a willingness to respond, not an ability to mimic well.

One of the factors affecting pronunciation mentioned by Piske et al. (2001) and discussed above in section 2, was gender. There are a number of interesting differences between the results of the female and male participants. Some notable variations are detailed in the table below.

\begin{tabular}{|c|c|c|c|c|c|c|c|}
\hline & Appearance & Anxiety & Boldness & $\begin{array}{c}\text { English } \\
\text { accent }\end{array}$ & $\begin{array}{c}\text { Mimicry } \\
\text { total }\end{array}$ & $\begin{array}{c}\text { Mimicry } \\
3 \mathrm{~s}\end{array}$ & $\begin{array}{c}\text { Mimicry } \\
\text { 0s }\end{array}$ \\
\hline $\begin{array}{c}\text { Male } \\
\text { mean } \\
(\mathrm{n}=6)\end{array}$ & 14.83 & 14.17 & 18.50 & 5.42 & 77.83 & 8.83 & 1.17 \\
\hline $\begin{array}{c}\text { Female } \\
\text { mean } \\
(\mathrm{n}=25)\end{array}$ & 17.56 & 17.60 & 15.68 & 5.49 & 67.96 & 8.44 & 5.16 \\
\hline
\end{tabular}

Table 5: Male/female differences

The stereotypes may be considered old-fashioned, but the data suggest that females are indeed more concerned about their appearance and less confident than their male counterparts. Nonetheless, the very similar accent means and number of $3 \mathrm{~s}$ scored suggest that pronunciation talent is distributed evenly between the sexes, with the obvious proviso that only 6 males took part and a larger sample is required to reinforce that statement. The fact that the mimicry total score is higher for the males reflects their willingness to give weak responses: the males remained silent (scored 0 ) on average only once, the females 5 times. Also, 3 out of 6 males responded to every input, only 3 out of 25 females did the same.

The primary aim of the study was to look for evidence of the Cecily effect. The first result to highlight is that, unlike in the pilot study, table 4 does show some correlation for the whole population between finding the language attractive and mimicry performance in that language. The same table shows a positive but very low correlation between feelings for English and English accent. The mean score for interest in appearance was 
17.03 (see table 1). 4 participants, however, scored 20 or more, all of them female. Their performances are detailed below in table 6 .

\begin{tabular}{|c|c|c|c|c|c|c|c|}
\hline & $\begin{array}{c}\text { Fondness } \\
\text { for Italian }\end{array}$ & $\begin{array}{c}\text { Fondness } \\
\text { for } \\
\text { Chinese }\end{array}$ & $\begin{array}{c}\text { Fondness } \\
\text { for Dutch }\end{array}$ & $\begin{array}{c}\text { Italian } \\
\text { mimicry }\end{array}$ & $\begin{array}{c}\text { Chinese } \\
\text { mimicry }\end{array}$ & $\begin{array}{c}\text { Dutch } \\
\text { mimicry }\end{array}$ & $\begin{array}{c}\text { English } \\
\text { accent }\end{array}$ \\
\hline $\begin{array}{c}\text { Cecilys } \\
\text { mean } \\
(\mathrm{n}=5)\end{array}$ & 12.40 & 6.80 & 9.40 & 24.40 & 24.60 & 24.20 & 6.30 \\
\hline $\begin{array}{c}\text { Non- } \\
\text { Cecilys } \\
\text { mean } \\
(\mathrm{n}=26)\end{array}$ & 11.08 & 7.65 & 8.50 & 22.42 & 24.42 & 21.85 & 5.32 \\
\hline
\end{tabular}

Table 6: The Cecilys

Although the differences between the two groups are not especially striking, the pattern which was expected from the pilot study has clearly developed. The top scorers for interest in appearance have above average English accent and would be expected to be better than average mimics. This expectation is met in the case of both Dutch and Italian, but their performance drops to just average for Chinese. The same is true for their appreciation of these languages: they have above average fondness for the two European languages but below average for Chinese. The fact that their relative performance drops for the least popular language suggests that this group is particularly sensitive to the unpleasantness of speaking a strange tongue, and that sensitivity may well be linked to their strong concern for their appearance. The variation in performance is not so high as to lead to definite conclusions but hypothesis 1, that the Cecily effect does operate on those with high levels of interest in appearance, has received some support from this study.

\section{Conclusions}

There can be little doubt, then, that personality and affective factors do influence the success of learners in developing a good accent in their L2, but the way in which that influence is exerted is complex and may vary considerably amongst individuals. As in the studies discussed above, no clear correlations were found between character traits and performance but a number of small steps have been taken towards a greater understanding of the impact of personality on pronunciation.

The results show that mimicry scores, when they include only examples of good mimicry, do correlate with foreign language accent scores and could, therefore, be used as a way of predicting likely success or failure in that field. Some evidence was found, however, to support the two hypotheses of the study, which illustrate the complex nature of the relationship between the two. Hypothesis 1, that the Cecily effect exists, is, to some extent supported: a high interest in one's own appearance does appear to interact 
with one's regard for the sound of the target language and, thus, affect performance. In addition to this, hypothesis 2 , that boldness influences mimicry scores but not accent, has also received a degree of support. This means that any test of mimicry which hoped to predict accent would need to take into account the willingness to take risks of the participants. This trend is illustrated best by the comparison of the male and female subjects, where the more confident males scored more highly for mimicry but almost exactly the same for good quality mimicry and accent as the females.

There is no room here for a full analysis of the performance of the tools. One worry from the pilot study was the low correlation of accent scores of the English native speakers at $r=0.51$. A change of second assessor produced an improvement in this regard with the two sets of marks correlating at $r=0.64$, which, however, still seems rather low. Another question over whether mimicry should, in fact, be treated as a more or less universal skill is left unanswered by the correlations between the scores for the different languages. They are: Italian/Chinese 0.39, Italian/Dutch 0.35, Dutch/Chinese 0.46. The idiosyncrasies of the Italian marker have already been discussed in section 6 above. For my own marks, however, the same correlations are: $0.55,0.33,0.61$. This raises the question of whether the use of native speaker markers for mimicry tests actually improves the quality of the data. Different raters obviously have different standards and there is also the question of experience in rating foreign accents: none of the three native-speaker raters is involved in teaching or assessment. I have generally found the use of native speakers to be considered essential by other researchers but remain unconvinced: the level of mimicry likely to be achieved after hearing a stimulus once does not need a native's ear to judge its quality.

To conclude, this study represents a very small step towards unravelling the complexity of the many factors which determine a learner's eventual level of accent in the target language. However, it is only by examining interactions one-by-one that we may hope to build a viable working model of the factors affecting foreign language pronunciation. To that end, the identification of the Cecily effect is one piece completed in a very large jigsaw puzzle.

\section{References}

Arnold, J. Ed. 1999. Affect in Language Learning. Cambridge: Cambridge University Press.

Baran-Łucarz. 2013. Phonetics learning anxiety - results of a preliminary study. Research in Language Vol. 11.1: 57-79. DOI: 10.2478/v10015-012-0005-9

Couper, G. 2003. The value of an explicit pronunciation syllabus in ESOL teaching. Prospect Vol. 18, No. 3 December 2003: 53-70

Couper, G. 2006. The short and long-term effects of pronunciation instruction. Prospect, 21, 46-66.

Carroll, J. B. 1965. The prediction of success in intensive foreign language training. In R.Glaser (ed.), Training, research and education, 87-136. New York: Wiley.

Derwing, T. M., \& Rossiter, M. J. 2003. The effects of pronunciation instruction on the accuracy, fluency, and complexity of L2-accented speech. Applied Language Learning, 13, 1-18. 
Dogil, G \& S.M. Reiterer, (eds) 2009. Language talent and brain activity. Berlin - New York : De Gruyter.

Dornyei, Z. 2010. The relationship between language aptitude and language learning motivation: individual differences from a dynamic systems perspective. In E. Macaro (ed.) 2010. Continuum companion to second-language acquisition, 247-267. London: Continuum.

Ganschow, L. \& R.L. Sparks. 1996. Anxiety about foreign language learning among high school women. The Modern Language Journal, 80, ii: 199-212

Hinton, M. 2012. The identification and testing of cognitive and affective factors in language learning aptitude with Polish students of English. Unpublished Ph.D thesis. University of Łódź.

Hinton, M. 2013. An Aptitude for Speech: The Importance of Mimicry Ability in Foreign Language Pronunciation. In Waniek-Klimczak, E. \& L. R. Shockey (eds), Teaching and Researching English Accents in Native and Non-native Speakers, Second Language Learning and Teaching (Ch. 8). Berlin Heidelberg: Springer-Verlag.

Hinton, M. In press. The Cecily Effect: a pilot study. In Social, Cultural and Affective Influences on Language Processes. University of Opole.

Horwitz, E.K., Horwitz, M.B \& J. Cope. 1986. Foreign Language Classroom Anxiety. The Modern Language Journal, 70, ii : 125-132

$\mathrm{Hu}, \mathrm{X} \&$ S.M. Reiterer. 2009. Personality and pronunciation talent. In Dogil, G \& S.M. Reiterer (eds) Language talent and brain activity, 97-130. Berlin - New York : De Gruyter.

Jilka, M. 2009. Talent and proficiency in language. In Dogil, G \& S.M. Reiterer, (eds) Language talent and brain activity, 1-16. Berlin - New York : De Gruyter.

Piske, T., I.R.A MacKay \& J. Flege. 2001. Factors affecting degree of foreign accent in an L2 : a review. Journal of Phonetics (2001) $29: 191-215$.

Purcell, E. \& R. Suter. 1980. Predictors of pronunciation accuracy: A re-examination. Language Learning. 30/2 : 271-87

Reiterer, S.M, X. Hu, M. Erb, G. Rota, D. Nardo, W. Grodd, S. Winkler \& H. Ackermann. 2011. Individual differences in audio-vocal speech imitation aptitude in late bilinguals: functional neuro-imaging and brain morphology. Frontiers in Psychology, Oct 2011, Volume 2, Article 271

Singleton, D. 2005. The Critical Period Hypothesis: a coat of many colours, International Review of Applied Linguistics (IRAL) 43 (2005): 269-285 


\section{Appendix A - Statements used in survey}

Importance of sound.

Pronunciation should be given more time on foreign language courses. (Question no:1)

Some languages sound nicer than others. 10

If someone has an unpleasant voice, I find it hard to concentrate on what he's saying.20

I am sensitive to the rhythm of different languages.21

I feel friendly towards someone if I like his voice. 25

Importance of appearance.

I spend a lot of money on clothes and cosmetics. 3

I don't like scruffy, untidy people. 18

I never leave the house without checking the mirror.11

I spend a lot of time each day on my appearance. 6

I don't like to be seen when I don't look my best. 26

Language anxiety

I would rather say nothing than say something stupid. 2

I feel nervous when I'm not sure how to pronounce a word. 5

I worry about how other people see me when I speak a foreign language. 8

I am happy with the sound of my voice when I speak English. 4 (Reverse score)

I always think other students speak English better than I do. 27

Regard for Polish

I think the Polish language is one of the most beautiful.7

I am proud to be Polish.16

I don't like to hear Polish mispronounced.9

I feel better speaking Polish than other languages.22

I like to hear other languages being spoken in Poland. 28 (Reverse score)

Openness to foreign cultures

I would like to travel in Asia.12

I would like to live in another country. 17

I am interested in all languages.13

I would like to learn a non-European language. 24

I would like to have friends from other cultures.33

Regard for English

I find the sound of English very attractive.14

Speaking English feels natural to me.19

I like to hear myself speaking English. 15

I want to sound like a native when I speak English.23

I would like to live in an English speaking country. 35 


\section{Boldness}

I like to take risks.6

I like to use new words, even if I'm not sure how to say them. 23

I don't like to answer questions in class unless I'm absolutely sure I know the right answer.3 (Reverse score)

I'm confident I can communicate in English, even in difficult situations.34

I like to join in conversations, even if I don't understand everything being said. 16

\section{Appendix B - Statements about attractiveness of input languages.}

I would like to speak this language.

I find the sound of this language attractive.

I like the rhythm of this language.

The speaker sounds funny to me.

\section{Appendix C - Reading passage for accent assessment}

The more he thought about how the unfortunate woman must be feeling, the more he laughed. Her entire day had been ruined; her husband would have no dinner this evening, perhaps he would beat her; more likely he would try to comfort her, 'It doesn't matter,' he would say, 'We can always buy some more.' He would, of course, completely misunderstand, because he had not been there, he had not seen the incident, felt the indignity of it, the utter hopelessness of standing in a crowded place, with cherryflavoured yoghurt splashed on his trousers, and being looked at, having a disaster in public. He would not have dropped the bag.

This picture of the weeping wife and consoling husband so tickled Claude that he did not hear the knock at his door over his own laughter. The visitor, however, was on sufficiently intimate terms with the resident to open the door himself and enter the room. The visitor coughed. Claude turned around sharply. (From Claude, by Martin Hinton) 